\title{
EMBEDDINGS INTO MONOTHETIC GROUPS
}

\author{
MICHAL DOUCHA
}

\begin{abstract}
We provide a very short elementary proof that every bounded separable metric group embeds into a monothetic bounded metric group, in such a way that the result of Morris and Pestov that every separable abelian topological group embeds into a monothetic group is an immediate corollary. We show that the boundedness assumption is essential.
\end{abstract}

In 2] Morris and Pestov prove that every separable abelian topological group embeds into a monothetic group (following their previous generalization of the Higman-NeumannNeumann theorem for topological groups from [1]). Since their proof is rather long and uses several non-elementary results we provide here a short elementary proof of their result which is actually a generalization: it is in the category of metric groups.

Theorem 0.1. Let $G$ be a separable abelian group with a bounded invariant metric d. Then $d$ extends to a (bounded by the same constant) metric $D$ on $G \oplus C$, where $C$ is a cyclic group which is dense in $G \oplus C$. In particular, $G$ embeds into a monothetic metric group.

Proof. Instead of metric, we shall work with the corresponding norm, i.e. the distance of an element from the group zero, which we shall still denote $d$, or $D$. That is, $d(g)$ denotes $d(g, 0)$. Without loss of generality, suppose that $d$ is bounded by 1 . Let $H=\left\{h_{n}: n \in \mathbb{N}\right\}$ be a countable dense subgroup of $G$ and let $\pi: \mathbb{N} \rightarrow \mathbb{N}^{2}$ be some bijection; we denote by $\pi(n)(1)$, resp. $\pi(n)(2)$, the respective coordinates of $\pi(n)$. Suppose the cyclic group $C$ is generated by some $c$. By induction, we shall construct a partial norm $D^{\prime}$ on $H \oplus C$ which extends $d$, i.e. a partial function satisfying $D^{\prime}(g)=0$ iff $g=0, D^{\prime}(g)=D^{\prime}(-g)$ and $D^{\prime}\left(g_{1}+\ldots+g_{n}\right) \leq$ $D^{\prime}\left(g_{1}\right)+\ldots+D^{\prime}\left(g_{n}\right)$, whenever the corresponding elements are in the domain of $D^{\prime}$. Moreover, we shall produce an increasing sequence $\left(k_{n}\right)_{n}$ such that $D^{\prime}\left(c^{k_{n}}-h_{\pi(n)(1)}\right) \leq 1 / \pi(n)(2)$. At the end, we may define $D$ by $D(x)=\min \left\{1, \inf \left\{\sum_{i=1}^{m} D^{\prime}\left(x_{i}\right): x=\sum_{i=1}^{m} x_{i},\left(x_{i}\right)_{i} \subseteq \operatorname{dom}\left(D^{\prime}\right)\right\}\right\}$, for $x \in H \oplus C$. Since $D^{\prime}$ was a partial norm, $D$ extends $D^{\prime}$, thus it extends $d$, and by the induction we will have guaranteed that $C$ is dense in $H \oplus C$.

At the first step of the induction, we set $D$ to be equal to $d$ on $H$, set $k_{1}=1$ and we define $D^{\prime}\left(c-h_{\pi(1)(1)}\right)=D^{\prime}\left(h_{\pi(1)(1)}-c\right)=1 / \pi(1)(2) . D^{\prime}$ is clearly a partial norm. Suppose we have done the first $n-1$ steps, found $k_{1}, \ldots, k_{n-1}$ and defined $D^{\prime}$ appropriately so that it is a partial norm. Let $\delta=\min \{1 / \pi(i)(2): i<n\}$ and let $k_{n}>k_{n-1}$ be arbitrary satisfying $k_{n}>k_{n-1} / \delta$. Then we set $D^{\prime}\left(c^{k_{n}}-h_{\pi(n)(1)}\right)=D^{\prime}\left(h_{\pi(n)(1)}-c^{k_{n}}\right)=1 / \pi(n)(2)$. We claim that $D^{\prime}$ is still a partial norm. Suppose on the contrary that the triangle inequality is broken, i.e. there are $x, x_{1}, \ldots, x_{n} \in \operatorname{dom}\left(D^{\prime}\right)$ such that $x=x_{1}+\ldots+x_{n}$ and $D^{\prime}(x)>D^{\prime}\left(x_{1}\right)+\ldots+D^{\prime}\left(x_{n}\right)$. We shall suppose that $x \in H$, that is the most important case and the other case is treated analogously. For any $z \in H \oplus C$, denote by $k(z)$ the unique integer such that $z$ can be written as $h \oplus c^{k(z)}$. Since $k(x)=0$, we must have $\sum_{i=1}^{n} k\left(x_{i}\right)=0$. For at least one $i \leq n$ we must have that $k\left(x_{i}\right)$ is $k_{n}$ or $-k_{n}$, since before the extension at the $n$-th step, $D^{\prime}$ was a partial norm. Also, since $H$ is abelian, we may suppose that for no $i, j \leq n$ we have $k\left(x_{i}\right)=-k\left(x_{j}\right) \neq 0$, since in that case $x_{i}+x_{j}=0$. Let $I \subseteq\left\{i \leq n: 0 \neq k\left(x_{i}\right) \neq k_{n}\right\}$. It follows that $\left|\sum_{i \in I} k\left(x_{i}\right)\right| \geq k_{n}$, so by definition of $k_{n}$ we have $|I|>1 / \delta$, so $\sum_{i \in I} D^{\prime}\left(x_{i}\right)>1$, a contradiction.

2010 Mathematics Subject Classification. 22A05, 54E35.

Key words and phrases. monothetic groups, metric groups, separable abelian groups. 
Notice that the numbers $\left(k_{n}\right)_{n}$ were chosen completely independently of the metric/norm, and the same sequence may be used for any metric/norm bounded by 1 . Secondly, notice that there is no change in the proof if we replace metric, resp. norm by pseudometric, resp. pseudonorm.

Corollary 0.2 (Morris, Pestov). Every separable abelian topological group G embeds into a monothetic group.

Proof. Let $H$ be a countable dense subgroup of $G$ and let $\left(\rho_{\alpha}\right)_{\alpha<\kappa}$ be a collection of pseudonorms bounded by 1 which give the topology of $G$, and $H$. By the previous proof we may extend each $\rho_{\alpha}$ on $H \oplus C$, where $C$ is cyclic, using the same numbers $\left(k_{n}\right)_{n}$ and $\pi: \mathbb{N} \rightarrow \mathbb{N}^{2}$. By either completing $H \oplus C$ with respect to these pseudonorms or extending the pseudonorms on $G \oplus C$ by amalgamation, we obtain a monothetic group to which $G$ embeds.

One may wonder why we assume boundedness of the metric/norm. It turns out it is essential. Consider $\ell_{1}^{2}$, the two-dimensional Banach space with $\ell_{1}$ norm, and let $H$ be its metric subgroup generated by the two basis elements $e_{1}, e_{2}$. Suppose there is a norm $D$ on $H \oplus C$, with $C$ cyclic, which extends the norm on $H$, and $C$ is dense in $H \oplus C$. Then there exist $n, m \in \mathbb{Z}$ such that $D\left(c^{n}-e_{1}\right)<1 / 2$ and $D\left(e_{2}-c^{m}\right)<1 / 2$. Then however $|m|+|n|=$ $D\left(-m \cdot e_{1}+n \cdot e_{2}\right)=D\left(c^{m n}-m \cdot e_{1}+n \cdot e_{2}-c^{n m}\right) \leq m D\left(c^{n}-e_{1}\right)+n D\left(e_{2}-c^{m}\right)<1 / 2(|m|+|n|)$, a contradiction.

Problem. Prove a metric version of the Higman-Neumann-Neumann theorem. Either for separable groups with general left-invariant metrics, or for groups with bi-invariant metric.

\section{REFERENCES}

[1] S. A. Morris and V. Pestov, A topological generalization of the Higman-Neumann-Neumann theorem, J. Group Theory 1 (1998), 181-187

[2] S. A. Morris, V. Pestov, Subgroups of monothetic groups, J. Group Theory 3 (2000), no. 4, 407-417

Institute of Mathematics, Academy of Sciences, Prague, Czech Republic

E-mail address: doucha@math.cas.cz 